\title{
Conséquences énergétiques de la sécheresse pour la production d'électricité
}

\author{
Daniel Ducloux \\ EDF - Service de la Production hydraulique
}

\section{Introduction}

La situation météorologique qui a sévi sur l'ensemble du pays pendant les deux premiers mois de l'année 1989 a entraîné des conséquences hydrologiques remarquables.

L'état des ressources hydroélectriques qui en a découlé a donc été tout à fait exceptionnel, pour cette période de l'année.

Pour Electricité de France, l'hydro-électricité représente une composante importante du parc de production électrique.

Une sécheresse, telle que celle que nous avons vécue l'hiver passé, si elle affecte bien entendu en premier lieu les performances et les capacités de la production hydraulique, aura également des répercussions importantes sur l'ensemble du parc de production, qui constitue un "système " dont toutes les composantes sont fortement interdépendantes.

\section{Analyse des conséquences énergétiques. Généralités}

\subsection{Exemple de l'hiver 1988-1989}

Afin d'examiner les conséquences énergétiques, au niveau du producteur d'électricité, d'une sécheresse sévère, la période de janvier et février 1989 est en effet un exemple significatif et intéressant, dans la mesure où :

- les principaux facteurs hydrauliques ou hydrologiques déterminant les possibilités du parc hydroélectrique ont tous été affectés ;

- le parc de production a désormais atteint une certaine "stabilité "; en effet, après une profonde mutation pendant les quinze dernières années, il est actuellement relativement proche de l'optimum, et l'on peut penser que, dans les années à venir, il variera peu par rapport à sa structure générale actuelle;

- cet épisode de sécheresse s'est produit dans la période

\section{Energy consequences of the drought on electricity production}

The consequences of the drought of the winter 1988-89 on the management of electricity production by EDF are both immediate and future.

In the short term, the shortage of electricity produced in plants that use water must be compensated for by use of more costly thermic means, in the long term the stock of seasonal lakes must be reconstituted for the following Winter.

Moreover, weak natural flow rates, in particular on the Rhone, lead to very tense management of the hydraulic chain and the head reservoirs, in order to ensure cooling of nuclear power stations.

Finally, in order to satisfy external requirements for next Summer (tourism, nautical sports) it is even more necessary to exploit certain establishments. 
normalement la plus défavorable, janvier et février, puisque c'est celle où la consommation électrique est habituellement la plus forte de l'année.

\subsection{Importance du contexte global du parc de production}

Il faut aussi considérer que les conséquences d'une sécheresse, sur le parc de production électrique, indépendamment de la structure de ce dernier et de l'époque où cette sécheresse se produit, seront également fonction de facteurs conjoncturels caractérisant l'état du moment du parc, tels que :

- la disponibilité des différents moyens de production;

- la disponibilité du réseau de transport d'énergie ;

- certaines inadaptations temporaires (travaux, particularités d'exploitation...);

- des aléas toujours possibles, au niveau de la production (avaries) ou de la consommation (vague de froid).

Ces facteurs complémentaires qui caractérisent le contexte général du parc de production, pourront bien entendu aggraver sensiblement les conséquences principales affectant directement le parc hydroélectrique.

\subsection{Analyse suivant les cycles décisionnels de la gestion}

La gestion d'un système aussi complexe que le parc de production d'EDF s'effectue nécessairement à partir d'études et de décisions prévisionnelles (pluriannuelles, annuelles, mensuelles, hebdomadaires, journalières) qui permettent de préparer, dans les meilleures conditions, la conduite à chaque instant et en temps réel du Système « Production - Transport - Consommation "; l'objectif essentiel étant d'assurer en permanence l'équilibre Production/Consommation, dans les meilleures conditions techniques et économiques.

Ces différents cycles décisionnels se déroulent en permanence, sont interactifs (chaque cycle prépare les données utilisées dans le cycle suivant) et, en affinant progressivement les paramètres de la gestion prévisionnelle, aboutissent à la conduite en temps réel.

Les incidences sur le système de production, d'un événement extérieur tel que la sécheresse, s'analysent donc tout naturellement selon ces cycles : prévisionnels et «temps réel ».

\subsection{L'hydroélectricité au sein du système "Production - Transport - Consommation "}

Pour le producteur d'électricité, le déficit des ressources hydrologiques affecte bien entendu en premier lieu, la production hydroélectrique.

Pour apprécier l'impact d'une sécheresse, il est donc intéressant de rappeler rapidement les caractéristiques et le rôle de la production hydraulique au sein du système global de production d'EDF.

Sur le plan quantitatif: $25 \%$ de la puissance électrique installée, $20 \%$ de l'énergie annuelle produite, ce qui en fait le $2^{\mathrm{e}}$ moyen, après le thermique nucléaire, de production électrique.

Sur le plan qualitatif : performances dynamiques importantes pour la régulation permanente du système ; grandes qualités cinétiques et réserve de puissance rapidement mobilisable, pour la sécurité du système ; fiabilité et disponibilité élevées.

Sur le plan économique : énergie bon marché, au niveau des coûts de production ; énergie souple et stockable, donc pouvant être valorisée par un placement optimum sur les périodes les plus difficiles (pointes de consommation journalière, périodes froides de l'hiver).

$\mathrm{Si}$, sur le plan quantitatif, le parc nucléaire a une importance prépondérante ( $70 \%$ de l'énergie produite), la production hydraulique en est le complément indispensable. En effet, la complémentarité du couple Nucléaire/Hydraulique, au niveau des performances et des rôles respectifs de ses deux composantes, assure la cohérence technique et économique du système global de production électrique.

\section{Etat du parc hydroélectrique. Principaux facteurs}

Les performances et les possibilités, immédiates ou à terme, du parc hydroélectrique, peuvent être caractérisées par les trois principaux facteurs hydrauliques suivants : le stock nival, le taux de remplissage des lacs, la productibilité hydraulique.

Nous indiquerons quelles étaient les valeurs de ces facteurs pendant la période de sécheresse, et comment se situaient ces valeurs réalisées par rapport aux valeurs moyennes habituelles pour cette période de l'année (janvier-février).

\subsection{Le stock nival}

Le volume de neige en altitude, et sa valeur en eau, conditionnent directement les apports hydrauliques futurs sur les bassins versants correspondants. On peut donc en déduire, pour les grands réservoirs de haute et moyenne altitude, le volume des apports d'eau qui sera stocké dans les barrages lors de la fusion nivale du printemps et de l'été.

Au début février, l'enneigement n'atteignait que la moitié ou le tiers de la valeur normale, selon les bassins ; il était notamment très faible dans les Alpes du Sud et quasiment nul dans le Massif Central. Cet état a ensuite légèrement évolué avec une réduction limitée du déficit (voir fig. 1 et 2, page suiv.).

En conséquence, les prévisions d'apports dans la plupart des grands lacs saisonniers étaient très en dessous des valeurs normales. Pour certains lacs notamment, les probabilités de remplissage lors de la fusion étaient faibles. 
Excepté quelques sites dans les Alpes du Nord où Tenneigement a été dépassé 8 à 9 années sur 10, il est actuellement le plus faible connu depuis 1953 dans les Alpes du Sud.

\begin{tabular}{|c|c|c|c|c|}
\hline \multirow{2}{*}{ Régions } & \multicolumn{4}{|c|}{$\begin{array}{l}\text { Valeurs relatives par rapport au stock normal de } \\
\text { fin dhiver pris comme référence commune : } 100\end{array}$} \\
\hline & $\begin{array}{l}\frac{\text { Slock normal }}{\text { au }} \\
\text { ler Février }\end{array}$ & $1 / 2 / 87$ & $1 / 2 / 88$ & $1 / 2 / 89$ \\
\hline ALPES DUNORD & 65 & 60 à 80 & 50 & $\frac{20 \text { aे } 40}{\text { (Mont Cenis } 10)}$ \\
\hline ALPES DUSUD & 70 & $\begin{array}{l}25 \text { a } 40 \\
\text { (Verdon } 10)\end{array}$ & $\begin{array}{l}40 \text { a } 60 \\
\text { (Verdon } 35)\end{array}$ & (Verdon $\frac{5 \text { à } 10}{n \text { negligeable) }}$ \\
\hline $\begin{array}{l}\text { PYRENEES } \\
\text { OCCIDENTALES }\end{array}$ & $\underline{70}$ & 60 à 70 & 50 & 20 \\
\hline $\begin{array}{l}\text { PYRENEES } \\
\text { CENTRALES }\end{array}$ & 70 & 70 & 60 & 20 \\
\hline HAUTE ARIEGE & 75 & 40 & 50 a 60 & 25 \\
\hline $\begin{array}{l}\text { PYRENEES } \\
\text { ORIENTALES }\end{array}$ & 70 & 50 & 40 a 50 & 35 \\
\hline
\end{tabular}

Valeur en eau des réserves de neige dans :

Dans le Jura :

. Ain à Vouglans: $10 \mathrm{hm}^{3}$

. Doubs au Chatelót $15 \mathrm{hm}^{3}$

. Valserine à Bellegarde $10 \mathrm{hm}^{3}$

1.

Dans lo Massif Central Ouest et Est : négllgeable

\section{PHYSIONOMIE DE L'ENNEIGEMENT AU 15 mars 1989}

Une légère réduction du déficit d'enneigement est constatée depuis le 1er mars dans les Alpes du Nord jusqu'a la haute vallée de la Durance (le stock ayant augmenté de 10 à 20 points) : état stationnaire dans les Pyrénées, mais réduction importante des stocks de neige de moyenne altitude (Massit Central et Jura).

\begin{tabular}{|c|c|c|c|c|}
\hline \multirow{2}{*}{ Régions } & \multicolumn{4}{|c|}{$\begin{array}{l}\text { Valeurs relatives par rapport au stock normal de } \\
\text { fin d'hiver pris comme réference commune : } 100\end{array}$} \\
\hline & $\begin{array}{c}\frac{\text { Slock normal }}{\text { au }} \\
15 \cdot \text { Mar }\end{array}$ & $15 / 3 / 87$ & $15 / 3 / 88$ & $15 / 3 / 89$ \\
\hline ALPESDUNORD & 90 & 100 & 100 & 45 a 65 \\
\hline ALPESDUSUD & $\underline{90}$ & 50 & $\begin{array}{l}75 \\
\text { (50 sur Verdon) }\end{array}$ & (20 $\operatorname{sur} \frac{40}{\text { Verdon) }}$ \\
\hline $\begin{array}{l}\text { PYRENEES } \\
\text { CENTRALESET } \\
\text { OCIDENTALES }\end{array}$ & 90 & 65 & $\begin{array}{c}80 \text { a } 100 \\
(100 \text { à } 140 \text { Arlège })\end{array}$ & 30 \\
\hline $\begin{array}{l}\text { PYRENEES } \\
\text { ORIENTALES }\end{array}$ & 90 & 65 & 90 & 25 a 30 \\
\hline
\end{tabular}

Au 15 mars le stock neigeux, en moyenne altitude, après fusion est estimé à :

Dans to Jura :

- Doubs au Chatelot : $20 \mathrm{hm}^{3}$

- Ain à Vouglans : $50 \mathrm{hm}^{3}$

- Valserine à Bellegarde : $25 \mathrm{hm}^{3}$

Dans to Massif Central :

2.

- Dordogne et Rhue : $10 \mathrm{hm}^{3}$

- Cère à Cantalès : $10 \mathrm{hm}^{3}$

- Maronne: $7 \mathrm{hm}^{3}$

- Truyere à Sarrans : $43 \mathrm{hm}^{3}$

\subsection{Le taux de remplissage des lacs}

L'exploitation optimale des réservoirs saisonniers consiste à utiliser l'énergie stockée correspondante, pendant les périodes les plus froides de l'hiver. Cette optimisation économique de l'eau conduit donc à vider les lacs saisonniers jusqu'à la fin de l'hiver, afin de pouvoir constituer ensuite le stock utilisable lors de l'hiver suivant, pendant la période d'apport du printemps et de l'été.

Par rapport à ce cycle annuel, durant janvier et février, les lacs ont eu un taux de remplissage très bas (voir fig. 3). A fin janvier, donc bien avant la fin de l'hiver, le taux de remplissage était déjà descendu à $28 \%$. C'est, à l'exception de 1949 , le taux le plus bas jamais enregistré pour ce mois.

Il faut noter, en outre, que, avant le début de la sécheresse, ce taux de remplissage était déjà bas pour l'époque. Ce déstockage très anticipé a été le résultat d'une très forte sollicitation des centrales hydrauliques, notamment de «lac», pendant les mois de novembre et décembre 1988, où des mouvements sociaux ont fortement perturbé le parc de production.

\subsection{La productibilité hydraulique}

La productibilité hydraulique d'une période donnée est la production hydraulique théoriquement possible, réalisée ou stockée (toutes les installations étant supposées en ordre de marche aux conditions nominales), compte tenu des apports naturels de cette période.

La productibilité observée d'une période est généralement comparée à la productibilité moyenne de la même période (calculée sur le plus grand nombre d'années possible). On exprime ainsi un coefficient de productibilité.

La productibilité est donc la traduction énergétique de l'hydraulicité.

Pendant la période de sécheresse étudiée, le coefficient de productibilité du mois de janvier a été de 0,52 ; c'est le plus faible jamais observé pour un mois de janvier. A l'échelon hebdomadaire, ce coefficient a été de 0,36 la semaine du 6 au 12 février.

Pendant les trois premières semaines de février, la productibilité journalière a varié entre 50 et $60 \mathrm{GWh}$, soit entre 30 et $40 \%$ de sa valeur moyenne pour cette période (voir fig. 4).

\section{Conséquences sur la gestion du parc de production}

Après avoir indiqué le poids spécifique de l'hydroélectricité au sein du système de production et létat des facteurs principaux qui la caractérisent à un moment donné, nous allons examiner maintenant les conséquences de la sécheresse, vécues lors des différents cycles de la gestion, pendant les mois de janvier et février 1989.

\subsection{Le contexte: situation initiale du parc}

Comme nous l'avons évoqué précédemment, les mouvements sociaux de novembre et décembre 1988, qui ont 
REMPLISSAGE DES RESERVOIRS SAISONNIERS EN 10a8/8

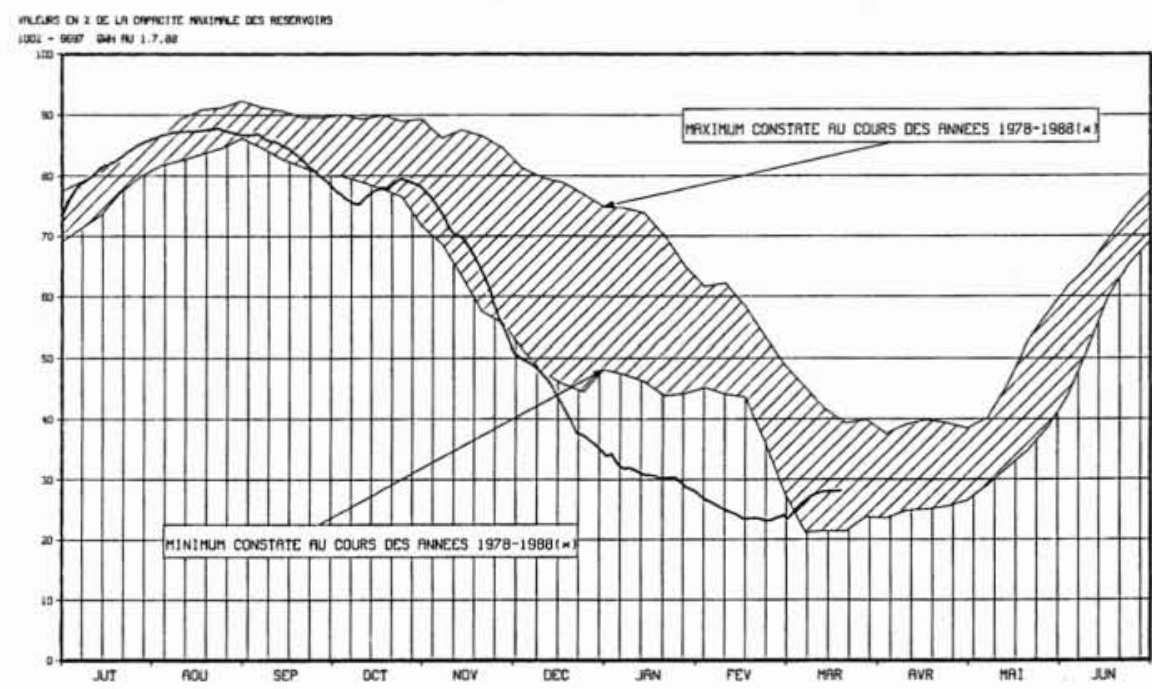

3.

PRODUCTIBILTES HYDRAULUOUES EN 1088/00

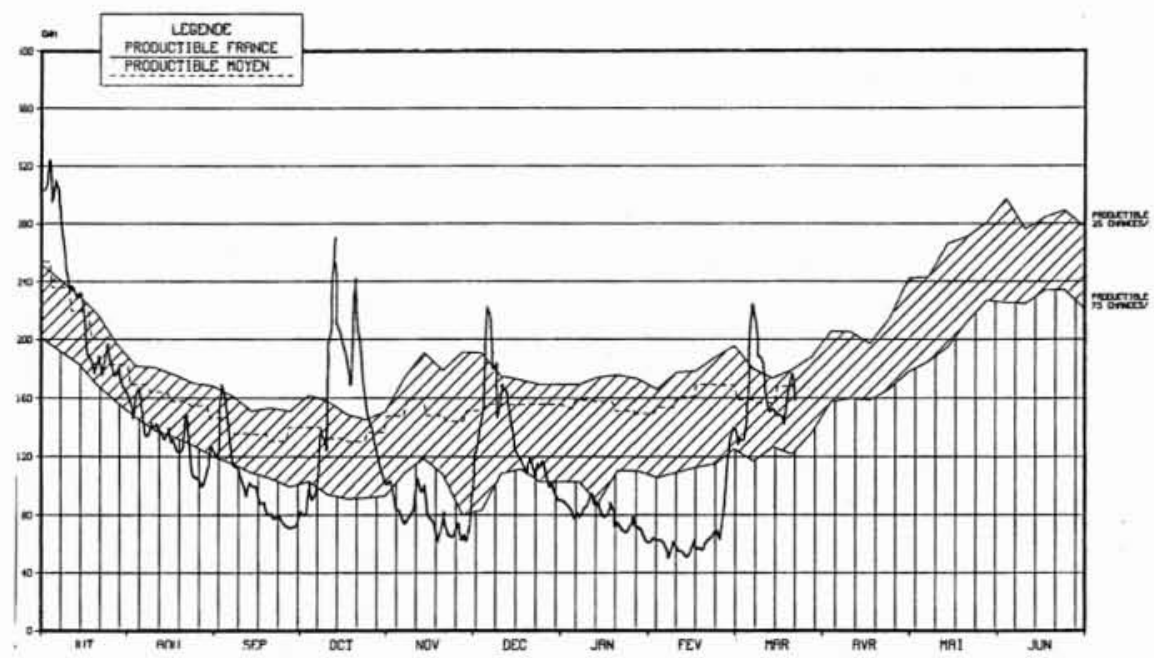

4.

affecté la production, et notamment la production thermique, ont eu trois conséquences essentielles sur l'état du parc au début de l'année 1989.

Disponibilité du parc thermique nucléaire: En janvierfévrier cette disponibilité est habituellement très forte; elle est normalement de 90 à $95 \%$ de la puissance installée ; il n'y a pratiquement pas d'entretien programmé.

En début de cette année 1989, compte tenu des retards pris sur des opérations d'entretien, cette disponibilité n'était que de $80 \%$ environ; huit tranches étaient arrêtées, au lieu de deux prévues, pendant le mois de janvier.
Stock de combustibles fossiles : Compte tenu du déficit de production nucléaire sur les deux derniers mois de 1988, la consommation de charbon et de fioul a été en forte augmentation ; pendant ces deux mois, il a été consommé $6 \mathrm{TWh}$ de charbon, soit deux fois plus que pendant les 10 mois précédents.

En conséquence, l'année 1989 a débuté avec un stock de combustibles fossiles beaucoup plus faible que prévu. Sur certains sites, le volume des stocks de charbon était même tout à fait préoccupant.

Stock hydraulique: Compte tenu de ce même déficit de production nucléaire en fin 1988 , le parc hydraulique, et 
BASSIN VERSANT , $250 \mathrm{~km} 2$ $\begin{array}{lll}\text { RESERVE UTILE, } & 236 & \mathrm{hm} \\ \text { SITUATION AU 1/3, } & 44 & \mathrm{hm}^{3}\end{array}$

NOTA. TOUTES PRISES EN SERVICE, EXCEPTES LES DERIVES DE L'ARC

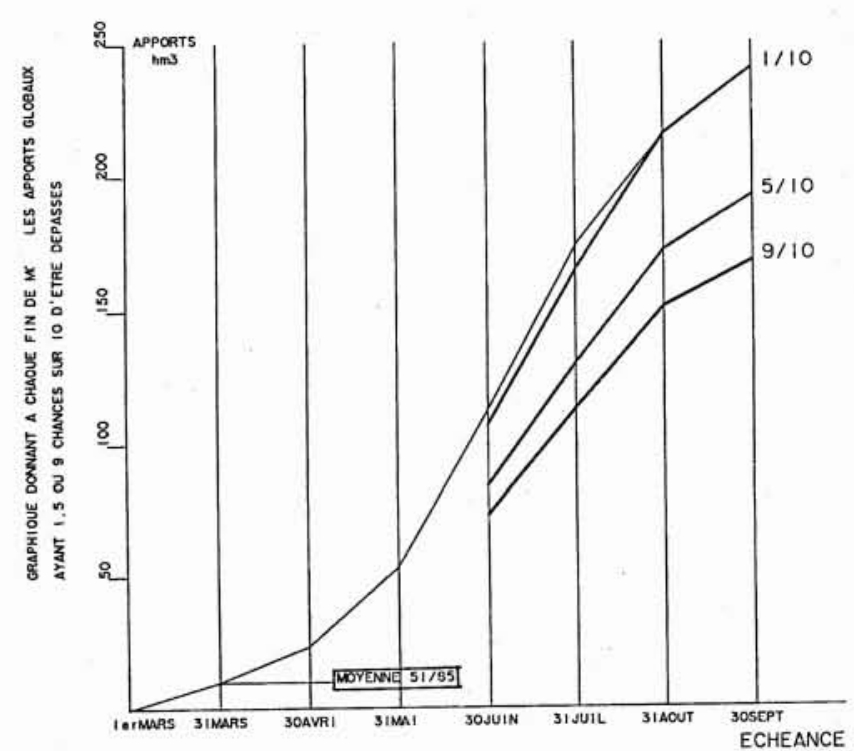

\begin{tabular}{|c|c|c|c|c|c|c|c|}
\hline $\begin{array}{c}\text { PROBABILITE } \\
\text { DE DEPASEER } \\
\text { LES VALERS } \\
\text { INOIOUEES }\end{array}$ & \multicolumn{6}{|c|}{ APPORIS PREVUS EN $\mathrm{Mm}$ POUR LA PERIODE ALLANT DU I/3 AU } \\
\hline & $30 / 6$ & $31 / 7$ & $31 / 8$ & $30 / 9$ & & & \\
\cline { 2 - 8 } & 107 & 165 & 215 & 240 & & & \\
$5 / 10$ & 84 & 130 & 172 & 192 & & & \\
$9 / 10$ & 73 & 113 & 151 & 168 & & & \\
\hline
\end{tabular}

5. Prévisions d'apports au $I^{\text {er }}$ mars 1989. Isère à Tignes.

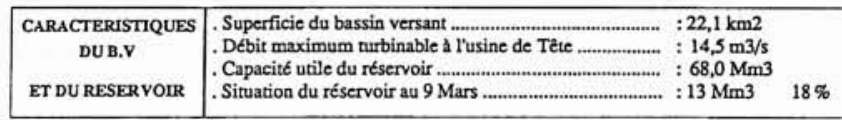

RPPORTS DU IER RURIL AU 31 JUILLET

\begin{tabular}{|c|c|c|}
\hline $\begin{array}{c}\text { PAOBABILTEDEDEPASSER } \\
\text { LES VALEURS INDIQUEES }\end{array}$ & $\begin{array}{c}\text { Apportsglobalux } \\
\text { Mm3 }\end{array}$ & $\begin{array}{c}\text { Nonstockables } \\
\text { Mm3 }\end{array}$ \\
\hline 10100 & 19,9 & 0 \\
\hline $25 / 100$ & 18,3 & 0 \\
\hline 50100 & 16,6 & 0 \\
\hline $75 / 100$ & 15 & 0 \\
\hline 50100 & 13,3 & 0 \\
\hline Moyenne $1956-1985$ & $\underline{23,2}$ & \\
\hline
\end{tabular}

6. Prévisions d'apport au 9 mars 1989. Lanoux H. C. notamment l'ensemble des centrales de lac, a été très fortement sollicité. Cela a entraîné en particulier un déstockage très important et anticipé des réservoirs saisonniers, de plus de $4 \mathrm{TWh}$ (environ $40 \%$ des réserves) pendant les deux derniers mois de l'année. Ce déstockage a conduit au très bas niveau des réservoirs dont nous avons parlé précédemment, avant même le début de la sécheresse.

Enfin, pour compléter le contexte général énergétique, deux points supplémentaires sont à noter, pour la période janvier-février 1989 :

- en moyennes hebdomadaires, les températures observées ont été supérieures aux températures normales pour cette époque. L'écart cumulé, par rapport à la température moyenne, était proche de $100^{\circ} \mathrm{C}$ - Jours, à fin février ;

- en conséquence, l'écart cumulé entre la consommation prévue et la consommation réalisée, pour ces deux premiers mois de l'année, a atteint environ 1,2 TWh, en déficit.

\subsection{Conséquences à long terme: quelques mois}

Il est nécessaire de constituer le stock hydraulique, pendant la fusion nivale du printemps et de l'été, afin d'aborder le prochain hiver 1989/1990 avec une réserve suffisante. En fonction du niveau des réservoirs, il faut donc maximiser leur probabilité de remplissage à la fin de l'été, suivant les apports prévisibles. Compte tenu du stock de neige, les apports prévisibles sont alors déterminés en y associant une probabilité.

Ainsi, au 15 février dernier, la plupart des lacs alpins et pyrénéens avaient environ une chance sur 10 d'atteindre ou de dépasser les apports normaux, pour la période jusqu'au 31 juillet (les apports normaux sont calculés en moyenne sur les réalisations des 30 dernières années).

Sur cette même période, les apports probables à neuf chances sur dix étaient de l'ordre, voire légèrement inférieurs, au creux du moment de certaines retenues comme Roselend, Tignes ou Serre-Ponçon, et nettement inférieurs pour d'autres retenues comme Cap de Long, Naguilhes et Lanoux, par exemple (voir fig. 5 et 6 ).

Pour ces raisons, à ce moment là il était donc nécessaire d'économiser fortement les lacs en les sollicitant le moins possible. Cette contrainte se cumulait donc avec celles découlant des faibles stocks de certaines centrales au charbon, et de la disponibilité limitée des centrales nucléaires.

Pour satisfaire l'équilibre Production/Consommation et pour constituer la marge supplémentaire de production nécessaire à la sécurité du système, il a été nécessaire alors 
de solliciter tous les moyens de production thermique disponibles, y compris les plus coûteux (centrales au fioul), en gérant au plus près les stocks de combustible.

Par ailleurs, l'exploitant dispose également de prévisions sur le coefficient de productibilité hydraulique des six mois à venir. Au 15 février, ce coefficient prévu était de 0,8 , avec une probabilité de 8 chances sur dix, pour l'ensemble "France", soit $28( \pm 5)$ TWh pour la période du $1^{\text {er }}$ mars au 31 août.

Les prévisions de remplissage des lacs d'une part et les prévisions de productibilité hydraulique d'autre part ont eu deux conséquences sur la gestion prévisionnelle du parc, vue du 15 mars :

- pour l'hiver prochain, on pouvait alors prévoir que le coût marginal de production correspondrait au coût du charbon pour une durée relativement importante, voire au coût du fioul ;

- pour réduire le coût marginal prévisionnel de production de certaines périodes difficiles, notamment en septembre, il a été nécessaire de réaménager le planning d'entretien du parc thermique, afin d'étaler et de répartir différemment les arrêts programmés ; le planning d'entretien du parc hydraulique pourrait également être modifié durant l'été.

\subsection{Conséquences à moyen terme: quelques semaines}

Pendant la période d'hiver, en particulier durant les deux premiers mois de l'année, la sécurité exige de se prémunir contre un aléa climatique d'ampleur importante, avec une faible probabilité d'aboutir à la défaillance du système; la défaillance consistant à ne pouvoir satisfaire toute la demande des consommateurs.

Cette disposition a bien entendu été prise pendant janvier et février dernier; la réserve énergétique correspondante devait permettre de faire face à une éventuelle vague de froid d'une durée de 2 semaines avec une température moyenne pondérée inférieure de $10{ }^{\circ} \mathrm{C}$ par rapport à la normale saisonnière.

Cette vague de froid choisie comme référence n'avait d'ailleurs rien de très exceptionnel, puisqu'elle équivalait à une température moyenne de l'ordre de $-5^{\circ} \mathrm{C}$, c'està-dire qu'elle était semblable à l'épisode climatique constaté, par exemple, en janvier 1987.

Cette contrainte supplémentaire nécessitait donc de ménager les stocks énergétiques nécessaires sous forme de charbon et d'hydraulique de lac. Elle a été prise en compte dans la gestion des moyens de production jusqu'au $1^{\text {er }}$ mars.

\subsection{Conséquences à court terme : en temps réel}

Le court terme se situe ici d'une part au niveau de la prévision journalière et d'autre part au niveau de la gestion en temps réel proprement dite, du système de production.

On prend alors en compte des paramètres d'exploitation qui peuvent varier très sensiblement dans un délai relati- vement court ; il s'agit notamment de la production au « fil de l'eau " qui est par définition faiblement modulable et non stockable.

Cette production représente plus de la moitié de la production hydraulique totale; son poids à court terme, lors d'une période de faible hydraulicité, est donc important, surtout lorsque l'hydraulique modulable (centrales de lacs) est faiblement utilisée, comme ce fut le cas en janvier et février 1989.

Globalement, pour le parc hydraulique, la production de ces deux mois a été inférieure de $64 \%$ par rapport à la production de la même période en 1988. Corrélativement, et pour les mêmes périodes, le parc thermique classique a vu sa production multipliée par plus de trois. A l'énergie hydraulique manquante a donc été substituée une énergie thermique classique (notamment au charbon) nettement plus coûteuse.

Ainsi, par rapport aux prévisions (faites en hydraulicité moyenne), le déficit de production hydraulique pour les deux mois de janvier et février 1989, a été de 4,8 TWh. Le surplus correspondant de production thermique classique a été de 3,6 TWh. La différence entre les deux valeurs, c'est-à-dire 1,2 TWh, correspond bien à la correction négative de la consommation, due à la clémence de la température, évoquée précédemment.

\subsection{Conséquences liées à la prise en compte des contraintes externes d'environnement}

Jusqu'ici nous avons donc examiné les conséquences directes de la sécheresse sur les paramètres internes qui caractérisent la structure, l'état et les performances du parc de production à un moment donné. La variation de ces paramètres, au fur et à mesure de leur connaissance, impose un réajustement de la gestion prévue, afin de toujours tendre vers l'optimum technique et économique.

En outre, certains paramètres supplémentaires peuvent être pris en compte pour gérer la ressource hydraulique, et notamment les stocks constitués dans certains réservoirs. Ces paramètres peuvent être occasionnels ou au contraire systématiques.

L'exemple le plus significatif et certainement le plus important du point de vue des contraintes qu'il entraîne, est le respect des cotes d'un certain nombre de retenues.

En effet, de nombreuses activités touristiques se sont développées autour des plans d'eau créés par les barrages de la Production hydraulique; dans certains cas, il existe des conventions entre EDF et les collectivités locales ou les municipalités.

Les besoins énergétiques restent bien entendu prioritaires et la garantie du respect de ces contraintes touristiques ne peut pas être absolue, mais EDF s'efforce, dans toute la mesure du possible, de satisfaire ces demandes externes.

Ainsi, pour une dizaine environ de grands lacs saisonniers, appartenant à des aménagements d'importance nationale, il existe des contraintes touristiques sous forme des cotes minimales à atteindre, pendant la période d'été, en général du $1^{\text {er }}$ juillet au 30 août (fig. 7). Ces contraintes 


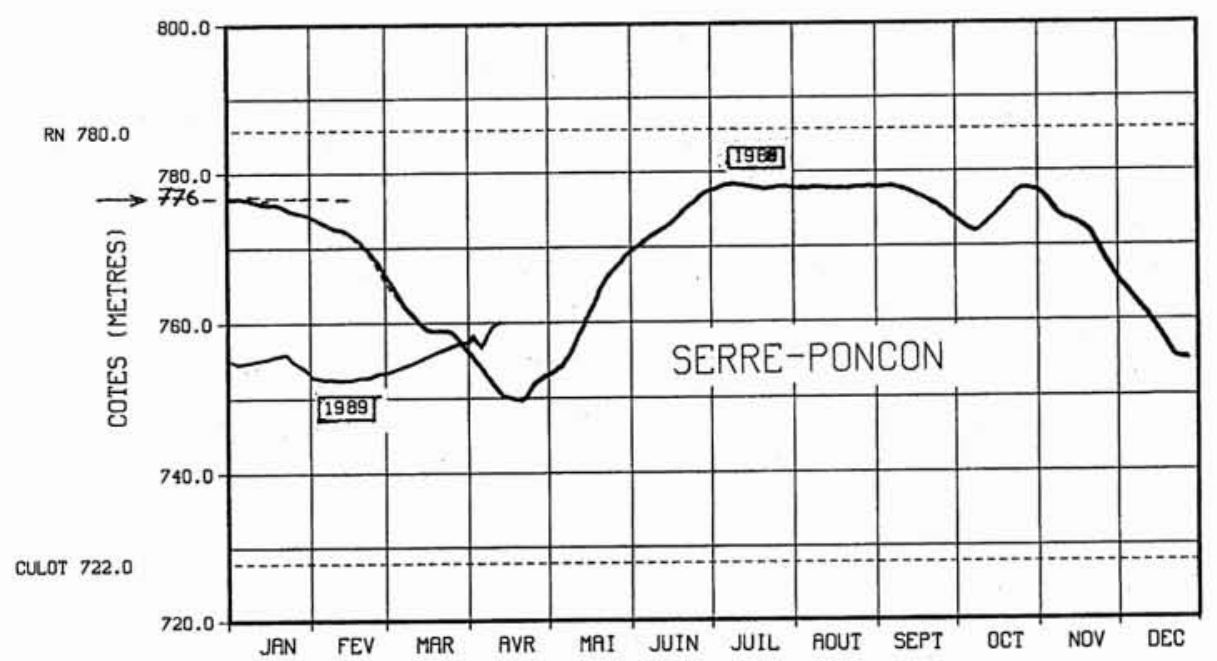

7.

sont intégrées dans les modèles de gestion de ces lacs, avec une probabilité de réussite prise généralement égale à 9 chances sur 10 .

Il est clair que ce paramètre supplémentaire, pour les besoins du tourisme, a limité encore plus fortement la gestion des lacs concernés pendant la sécheresse de ce début d'année. L'incidence sur l'exploitation a été particulièrement importante pour les retenues de Pareloup (aménagement du Pouget), de Vouglans (sur l'Ain), de Serre-Ponçon (sur la Durance) et de Ste-Croix (sur le Verdon). Ces aménagements ont dû être très peu sollicités et certains sont restés disponibles, à l'arrêt, pendant plusieurs semaines.

C'est-à-dire que, pendant cette période, en cas de nécessité, des moyens très coûteux comme des groupes de production au fioul, voire même des turbines à gaz, auraient été sollicités avant les trois aménagements hydrauliques cités.

Il faut cependant remarquer que la plupart des lacs comportant des contraintes touristiques sont situés en moyenne altitude (inférieure à 1000 mètres), leurs apports sont d'un régime mixte pluvio-nival (Serre-Ponçon) ou essentiellement pluvial (Bort-les-Orgues). Leur remplissage pourrait donc encore s'améliorer par des précipitations de printemps.

\subsection{Conséquence spécifique : gestion des débits du Rhône}

Enfin, un problème plus particulier est apparu lors de la sécheresse; il a pu être résolu rapidement au bout de quelques jours, mais, là aussi, les conditions hydrologiques ont eu des conséquences directes sur l'exploitation et la conduite de certains aménagements hydrauliques, et des mesures spécifiques ont dû être mises en œuvre rapidement.
Il s'agit des conséquences dues au faible débit du Rhône, sur les conditions de réfrigération de la centrale nucléaire de St-Alban, à l'aval de Lyon.

Cette centrale comporte deux tranches de $1300 \mathrm{MW}$, non modulables actuellement. La réfrigération est assurée par l'eau du Rhône, en circuit ouvert et sans aéroréfrigérant. Les contraintes de rejet thermique sont définies par un arrêté, selon trois paramètres :

- température de rejet $\left(<30^{\circ} \mathrm{C}\right.$ et $<32{ }^{\circ} \mathrm{C}$ du $1 / 6$ au $30 / 9)$,

- température aval après mélange $\left(<28^{\circ} \mathrm{C}\right.$ et $<30^{\circ} \mathrm{C}$ pendant $168 \mathrm{~h}$ par an),

- échauffement maximum entre l'amont et l'aval (de $1,5^{\circ} \mathrm{C}$ à $4{ }^{\circ} \mathrm{C}$, selon les époques).

Pour pouvoir respecter ces paramètres, les débits du Rhône doivent être maintenus au-dessus de certaines valeurs.

Les seuils de températures imposés ne doivent pas être dépassés en valeur moyenne sur une période de 1 heure. Dans la pratique, on s'attache à rester en permanence sous les seuils, en valeur instantanée.

La centrale nucléaire de St-Alban est située entre les centrales hydrauliques de Vaugris à l'amont et de Sablons à l'aval. Ces aménagements hydrauliques au fil de l'eau sont surveillés et conduits à distance par le poste de surveillance hydraulique de Châteauneuf-du-Rhône; le principe de la conduite consiste à réguler le niveau des plans d'eau amont (contraintes de navigation), avec une oscillation naturelle du débit de $\pm 5 \%$ autour de la valeur de consigne (périodicité d'environ 8 heures).

Au début du mois de février, le débit naturel du Rhône, au droit de St-Alban, était faible et de l'ordre de $320 \mathrm{~m}^{3} / \mathrm{s}$.

La contrainte d'échauffement des rejets de St-Alban est alors de $3{ }^{\circ} \mathrm{C}$ maximum (du $1 / 2$ au $31 / 5$ ) ; le débit corres- 
pondant minimum nécessaire du Rhône doit être alors de $390 \mathrm{~m}^{3} / \mathrm{s}$.

Une première nécessité est de remonter le débit naturel du Rhône en faisant des éclusées hebdomadaires et des déstockages des retenues hydrauliques amont de Génissiat sur le Rhône et de Vouglans sur l'Ain.

La deuxième nécessité est de caler le débit des usines hydrauliques de Vaugris et de Sablons à une valeur de $410 \mathrm{~m}^{3} / \mathrm{s}$, compte tenu des oscillations de $\pm 20 \mathrm{~m}^{3} / \mathrm{s}$, pour ne jamais descendre en dessous de $390 \mathrm{~m}^{3} / \mathrm{s}$.

Une telle exploitation pendant plusieurs semaines conduisait à un déstockage très important de Vouglans et à une forte probabilité de ne pas respecter la contrainte touristique de cette retenue, dont nous avons parlé précédemment. La solution adoptée a alors consisté à prendre des dispositions spécifiques au niveau de la conduite des usines hydrauliques :

- d'une part, par une exploitation affinée des ouvrages de restitution des réservoirs amont (Sault Brenaz sur le Rhône et Allement sur l'Ain);

- et surtout par un ajustement de la conduite automatique des usines hydrauliques du Rhône de Pierre Bénite, Vaugris et Sablons, à proximité de St-Alban.

Ce mode d'exploitation temporaire a consisté à intervenir directement sur les calculateurs de conduite de ces usines, afin de passer à un pilotage par consigne de débit minimum (celui correspondant à la contrainte), en surveillant très attentivement et en permanence le niveau des biefs, afin de ne pas perturber la navigation.

Cela revenait à réguler très finement le débit, calé sur la valeur minimale demandée $\left(390 \mathrm{~m}^{3} / \mathrm{s}\right.$ à Sablons), et par conséquent à économiser le surdébit correspondant aux oscillations naturelles, en exploitation normale.

La suppression de ce surdébit, lequel était obtenu essentiellement à partir du déstockage de Vouglans, a permis de faire revenir l'exploitation de cette retenue dans une zone plus "confortable", bien que déjà fort contrainte par ailleurs (cote touristique).

L'inconvénient essentiel d'un tel type d'exploitation se situe au niveau des interventions du personnel de surveillance et de conduite qui deviennent beaucoup plus importantes.

\subsection{La valeur économique de l'eau}

Après cette longue liste des perturbations que la sécheresse a apportées à l'exploitation du parc de production d'EDF, on constate que toutes ces conséquences sont fortement interdépendantes et souvent cumulatives. En effet, la gestion doit tenir compte de toutes ces contraintes, simultanément, même si elles se manifestent à des niveaux divers, et dans des cycles temporels de gestion différents.

L'indicateur le plus synthétique et le plus significatif qui illustre toutes ces difficultés d'exploitation est la valeur économique que les modèles de gestion énergétiques donnent à l'eau, à un moment donné.

La gestion énergétique de l'eau est complexe, elle utilise des critères dont certains relèvent de la prévision (apports, productibilité, consommation, etc...). La gestion économique des lacs conduit notamment à effectuer un arbitrage permanent entre l'utilisation immédiate de l'eau selon les conditions connues du moment, et l'utilisation ultérieure selon les conditions futures calculées en espérance.

$\mathrm{Au}$ bout de la chaîne décisionnelle, l'exploitant chargé de la conduite du système de production dispose alors d'indicateurs économiques synthétiques : les coûts d'ordre. Ces coûts d'ordre intègrent de très nombreux paramètres qui reflètent les caractéristiques, les performances, les disponibilités et les contraintes de tous ordres, des différents moyens de production. Pour une période donnée, ces coûts d'ordre permettent à l'exploitant, afin de satisfaire la consommation, de solliciter les différents moyens de production, en commençant par les moyens les plus économiques, et en empilant les centrales nécessaires, par coûts croissants. Pour les centrales hydrauliques, ces coûts d'ordre reflètent donc, entre autres éléments, et à un moment donné, la rareté de la ressource hydraulique, à court et à long termes, et l'opportunité de l'utiliser à l'instant de la décision.

Ainsi, de façon très résumée, on peut dire que dans la $2^{\mathrm{c}}$ partie du mois de février, tous les grands lacs saisonniers avaient des coûts d'ordre égaux et souvent supérieurs aux coûts des centrales thermiques au fioul. Après le 15 mars, compte tenu d'une amélioration des conditions de l'exploitation à court terme (disponibilité du thermique, productibilité hydraulique, abandon de la probabilité d'une vague de froid, etc...), les coûts d'ordre hydraulique ont sensiblement diminué vers des coûts proches de ceux du charbon.

Comme nous l'avons déjà évoqué, pendant toute cette période et au moins jusqu'à la fin mars, trois lacs saisonniers notamment étaient fortement contraints, en particulier à cause du respect de la cote "touristique" d'été : Pareloup, Serre-Ponçon et Ste-Croix ; leurs coûts d'ordre étaient au niveau de celui des turbines à gaz, c'est-à-dire au niveau des moyens de production les plus coûteux, donc en principe utilisables en cas d'ultime nécessité.

\section{Conclusion}

A la fin du mois de février et au début du mois de mars, on a observé une brutale remontée de la productibilité hydraulique avec une importante hausse des débits, notamment sur le Rhône.

A la mi-mars, on retrouvait donc des valeurs beaucoup plus conformes aux moyennes habituelles, en ce qui concerne :

- le coefficient de productibilité, proche de 1 ,

- le remplissage des réservoirs en augmentation, proche de $30 \%$, et dans une zone plus normale pour l'époque.

A court terme, la production hydraulique au fil de l'eau remontait fortement; ainsi, dans la première semaine de mars, la puissance disponible sur les centrales correspondantes atteignait $4000 \mathrm{MW}$, soit le double de ce qu'elle était vers le 20 février. 
Par ailleurs, les débits plus soutenus du Rhône supprimaient les contraintes de réfrigération nucléaire.

Enfin, l'éventualité d'une importante vague de froid n'était plus prise en compte à partir du début mars.

Ainsi, l'amélioration de certaines conditions d'exploitation du parc thermique (disponibilité nucléaire, stocks de combustibles fossiles) d'une part, et l'assouplissement des contraintes hydrauliques immédiates (productibilité, réserve de sécurité dans les lacs) d'autre part, permettaient donc, au cours du mois de mars, un retour vers des conditions d'exploitation plus confortables et plus normales. En particulier, les coûts d'ordre, c'est-à-dire en quelque sorte la valeur d'usage, d'un certain nombre de centrales hydrauliques de lac, revenaient à des valeurs permettant une sollicitation nettement plus soutenue de ces aménagements.

Cependant, il faut noter que, pour le plus long terme, la faiblesse des prévisions d'apport due au déficit neigeux, et des prévisions de productibilité pour les 6 mois à venir, maintenait les difficultés évoquées précédemment en ce qui concerne la constitution du stock énergétique de certains lacs saisonniers, et la satisfaction des demandes liées au tourisme sur certaines retenues.
Notamment, si une sécheresse sévère se manifestait à nouveau et se poursuivait jusqu'à l'automne, les difficultés évoquées pour aborder l'hiver prochain seraient notablement aggravées.

Pendant ces deux mois de sécheresse, qui ont affecté profondément les possibilités du parc hydraulique et malgré un certain nombre d'éléments aggravants en ce qui concerne la situation du parc avant même le début de cette sécheresse, on peut constater qu'à aucun moment la sécurité du système de production et de transport n'a été menacée.

Les conséquences se sont traduites essentiellement par des décisions et des modes d'exploitation inhabituels.

Cette situation, bien qu'exceptionnelle, a été prise en compte et gérée par les méthodes et les modèles habituellement utilisés par EDF pour exploiter et conduire le système dont elle a la charge.

L'optimisation technique et économique a donc continué d'être assurée, même s'il en a découlé un surcoût d'exploitation inévitable, face à un tel aléa de la nature. 